\title{
Improved Native Tissue Ultrastructure Investigations by High Pressure Freezing and Cryo-SEM
}

\author{
A. Kaech, ${ }^{*}$ H. Gross, ${ }^{* *}$ R. Wepf,*** \\ * Bal-tec AG, Neugruet 7, 9496 Balzers, Principality of Liechtenstein \\ ** Electron Microscopy Center of ETH Zurich (EMEZ), 8092 Zurich, Switzerland \\ *** R\&D Beiersdorf AG, Unnastrasse 48, Hamburg, Germany
}

High pressure freezing is the best method of choice to preserve the ultrastructure of soft condensed and hydrated matter (e.g. biological specimens, polymers). In combination with Cryo-FESEM it becomes the fastest technique to investigate the ultrastructure of such samples in a native or fully hydrated state. The entire processing of the specimen from object extraction to immobilization, surface preparation, coating, and imaging can be done in less than one hour with the right experimental setup.

The most critical steps, once the specimens are adequately frozen, are handling and the transfer of the frozen sample for preparation. The specimen carriers used for high pressure freezing (containing the frozen sample) have to be mechanically fixed to a specimen stage equipped with a clamp allowing fracturing, partial freeze-drying and transport between the different devices. This is significantly facilitated with a newly developed, versatile specimen stage.

Exposure to the air (moisture) and hence contamination must be avoided as well during the transfer of the specimen from liquid nitrogen to the high vacuum preparation device as during the transfer from the preparation device to the SEM. This is achieved with the Bal-Tec VCT 100 high-vacuum cryo loading and transfer system. The specimen is withdrawn from liquid nitrogen in the loading box into a cold, dry nitrogen gas atmosphere in the transfer shuttle equipped with a cold trap. The shuttle valve is closed and the protected specimen is transferred to high vacuum e.g. into a precooled freeze-fracturing and coating device. The transfer between the preparation devices and the CryoSEM is performed under high vacuum, controlled temperature conditions and protected by a cold trap [1]. Consequently, the specimen is never exposed to ambient conditions or high temperature.

Native mouse tissue was excised using a commercial biopsy gun. Biopsies were moved from the biopsy needle indentation directly into the recess of an aluminum specimen carrier filled with hexadecene. The sandwich was completed and frozen immediately. The sandwich was composed of two aluminum specimen carriers with $100 \mu \mathrm{m}$ cavities facing each other. After freezing, the sandwich was opened and the carrier containing the tissue was attached to the new specimen holder. The specimens were fractured with a cold hard metal knife in the freeze-fracturing device, partially freeze dried, coated and subsequently transferred to the SEM equipped with a temperature controlled cryo (cold) stage.

High resolution images of mouse organs were obtained, allowing new insights of the well preserved ultrastructure, e.g. protein patterns of membrane fracture faces, arrangement and dimensions of organelles and membranes in cross-fractured areas. The high throughput of this method [2] is ideally bridging the gab between CLSM and TEM investigations in space and in time. 


\section{References}

[1] M. Ritter et al., Microsc. Microanal. 5 (Suppl 2) (1999) 424.

[2] T. Richter et al., Skin Pharmacol. Physiol. 17 (2004) 246. 